V.V. Botvinyeva ${ }^{1}$, O.B. Gordeeva ${ }^{1}$, A.S. Potapov $^{1}$, L.S. Namazova-Baranova ${ }^{1,2,3}$, I.V. Zubkova ${ }^{1}$, A.O. Anushenko ${ }^{1}$, I.Z. Dzhgarkava ${ }^{1}$, M.A. Soloshenko ${ }^{1}$, A.K. Gevorkyan ${ }^{1,2}$

${ }^{1}$ Scientific Center of Children's Health, Moscow, Russian Federation

${ }^{2}$ First Sechenov Moscow State Medical University, Russian Federation

${ }^{3}$ Pirogov Russian National Medical Research University, Moscow, Russian Federation

\title{
Assessment of inflammatory response indicators and peripheral hemopoiesis at non- specific ulcerative colitis in children
}

\section{Author affiliation:}

Botvin'eva Viktoriya Vladimirovna, PhD, professor, chief research scientist at the Laboratory of Experimental Immunology and Virology of Research Institute of Pediatrics of Scientific Center of Children's Health

Address: 2/1, Lomonosovskii Ave., 119991, tel.: +7 (495) 967-14-20, e-mail: botvineva@nczd.ru

The article presents the analysis of laboratory tests, which indicate the response of the body to systemic inflammation. The revealed interconnection of these parameters with peripheral hematosis alterations at chronic inflammatory diseases will allow objectively approaching the diagnostics of functional disorders at non-specific ulcerative colitis in children. The authors attempted to develop new approaches to the analysis of laboratory indicators, which will help to evaluate individual dynamics of processes at the systemic inflammatory response of the body and select adequate therapy of the primary disease.

Keywords: systemic inflammatory response, ulcerative colitis, peripheral blood parameters, inflammatory markers.

\section{Introduction}

The study of interconnections of various blood parameters, such as serum markers, which reflect inflammatory alterations in the body, and peripheral hemopoiesis parameters is of considerable interest. It is common knowledge that severity of inflammation course at chronic diseases manifests itself with the degree of malfunction of the immune and hematopoietic systems in children. Some scientists claim that immune complex disorder of various organs and systems [1, 2] prevalent in the lesion focus of populations of anti-inflammatory cytokines is a trigger; this may cause increased permeability and malfunction of endothelium. These pathophysiological alterations support chronic inflammatory reaction characterized by the increased level of blood inflammatory response markers. Practicing physicians have to rely on a range of laboratory parameters, which are not capable of reflecting malfunction mechanisms of the studied system when separate. That is why analysis of blood as of a multitude of interconnected elements may promote better understanding of regulation mechanisms, appraisal of clinical laboratory data and functional capabilities [3]. Interconnection of alterations of serum parameters reflecting inflammatory reaction of the body (acute phase markers and iron metabolism parameters) and alterations revealed in children with nonspecific ulcerative colitis (NUC) by clinical and endoscopic examination remains insufficiently explored. The difficulty is that most of these parameters feature low specificity and are, therefore, insufficiently evidential for diagnostics (due to the lack of complex approach to their evaluation).

\section{Patients and methods}

We examined 78 children of 3-17 years of age, 38 of them had ulcerative colitis. The control group was comprised of 40 healthy children. Boys/girls ratio was 1:1. The average age of patients was 11.84 years. All children were undergoing inpatient examination and treatment at the gastroenterology department with hepatology group of the FSBI "SCCH" with diagnosis "Ulcerative colitis" (UC). Duration of the disease from diagnosis to the start of the trial varied 
from 6 months to 12 years. All patients were divided into groups according to the disease activity (low, moderate, high). UL activity was assessed as per the pediatric nonspecific ulcerative colitis activity index (PUCAI) [4] based on non-invasive evaluation of 6 clinical parameters: abdominal pain, rectal hemorrhage, stool consistency, number of defecations per day, number of nocturnal defecations and motion activity level. The index highly correlates with laboratory and endoscopic activity of the disease. All children underwent analysis of the blood stabilized with EDTA (calcium-sodium ethylenediaminetetraacete) using an automatic hematologic analyzer Sysmex 2000 I (Japan) to calculate parameters reflecting peripheral hemopoiesis (erythrocytes, erythrocyte indices, hemoglobin, leukocytes, platelets, platelet indices) [5]. Inflammatory markers and iron metabolism parameters (C-reactive protein (CRP), transferrin, iron) were studied in blood serum using a biochemical analyzer Unicell DxC 600 BD (USA). Blood serum was also analyzed with the help of immunochemical analyzer Access 2 (BD, USA) in order to determine ferritin and interleukin (IL) 6 concentration. Immune-enzyme analysis was conducted to determine hepcidin level (ELISA, IBL). The obtained data were processed with the help of statistics software SPSS 20.0 using a non-parametric method of analysis of variance. Null or alternative hypothesis of parameter spread was adopted according to the analysis results. Kruskal-Wallis test was used to compare 2 separate groups. ROC-analysis was used to evaluate sensitivity and specificity of laboratory tests (pic. 1).

Pic. 1. Trial design

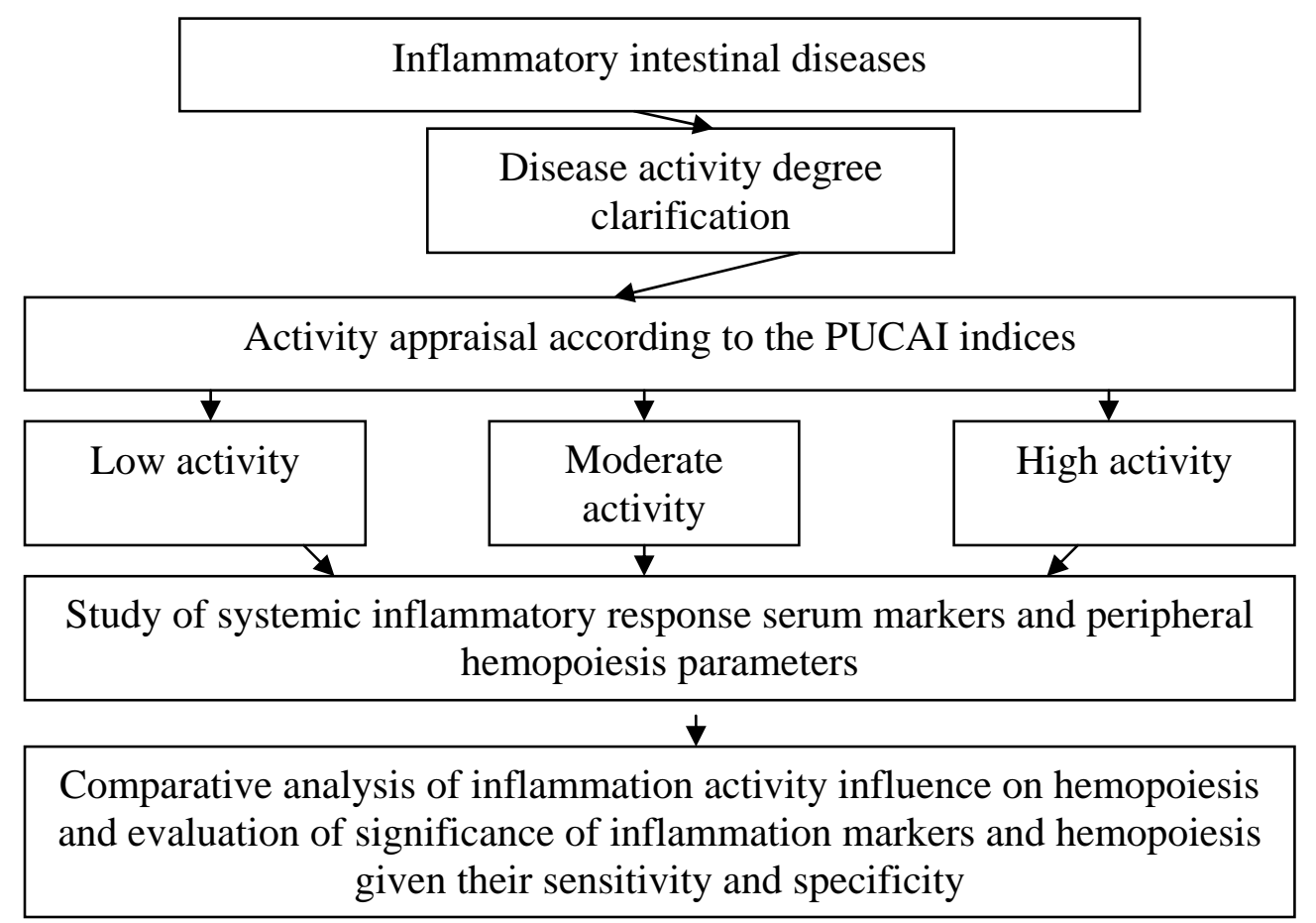

\section{Results}

We analyzed peripheral blood parameters, such as erythrocytes, MCV (mean corpuscular volume), $\mathrm{MCH}$ (mean corpuscular hemoglobin), MCHC (mean corpuscular hemoglobin concentration), hemoglobin level, number of leukocytes and platelets together with serum parameters changing in the setting of inflammatory reaction (CRP, IL 6, ferritin, iron, transferrin, hepcidin - a new laboratory parameter characterizing response of the body to inflammation and affecting iron metabolism) [6, 7]. According to the given tables (tb. 1), there is response of the hematopoietic system in case of inflammatory reaction of the body in the setting of ulcerative colitis in the form of moderate increase in the number of leukocytes, simultaneous reduction in the red blood indices (MCV, MCH, MCHC) characterizing functional status of hemopoiesis and 
insignificant alteration of the number of blood cells in comparison with healthy children $(\mathrm{p}<0.05)$. MCHC is the most significant erythrocyte index (pic. 2). Number of platelets and erythrocytes did not significantly differ from the corresponding indices in the group of healthy children ( $\mathrm{p}>0.05)$.

Table 1. Medians of the blood parameters under study at nonspecific ulcerative colitis in children.

\begin{tabular}{|c|c|c|c|c|c|}
\hline $\begin{array}{l}\text { Laboratory blood } \\
\text { parameters }\end{array}$ & $\begin{array}{l}\text { Nonspecific } \\
\text { degree }\end{array}$ & ulcerative & activity & $\begin{array}{l}\text { Control } \\
\text { group }\end{array}$ & $\begin{array}{l}\text { Significance } \\
\text { level } p\end{array}$ \\
\hline $95 \%$ CI & Low activity & $\begin{array}{l}\text { Moderate } \\
\text { activity }\end{array}$ & High activity & & \\
\hline CRP, mg/l & $\begin{array}{l}0.1 \\
19.88)\end{array}$ & $2.7(0.1-20.1)$ & $\begin{array}{l}6.8 \\
18.7)\end{array}$ & $0.1(0.1-1.0)$ & $0.025 *$ \\
\hline Ferritin, ng/ml & $\begin{array}{l}27.4 \\
131.2)\end{array}$ & $\begin{array}{l}30.2 \\
250.5)\end{array}$ & $\begin{array}{l}20.4 \\
110.1)\end{array}$ & $\begin{array}{ll}23.5 & (16.6- \\
33.9) & \end{array}$ & 0.98 \\
\hline IL 6, pg/ml & $\begin{array}{ll}3.63 & (0.98- \\
16.9) & \\
\end{array}$ & $\begin{array}{ll}5.56 & (2.53- \\
8.75) & \\
\end{array}$ & $\begin{array}{ll}6.37 \\
18.21)\end{array}$ & $2.2(1.5-3.5)$ & $0.000 *$ \\
\hline Hepcidin, ng/ml & $\begin{array}{l}118.21 \\
607.9)\end{array}$ & $\begin{array}{l}176 \\
723)\end{array}$ & $\begin{array}{l}198.9 \\
1,000)\end{array}$ & $\begin{array}{ll}36.5 & (0.1- \\
76) & \end{array}$ & $0.000 *$ \\
\hline Iron, mcmol/l & $\begin{array}{l}14.8 \quad(3.2- \\
29.4)\end{array}$ & $6.8(2.1-18.7)$ & 6.55 (3.4-9) & $\begin{array}{ll}13.9 & (9.2- \\
23.6) & \\
\end{array}$ & $0.001 *$ \\
\hline Transferrin, nmol/l & $\begin{array}{l}276 \quad \text { (216- } \\
336)\end{array}$ & $\begin{array}{l}282 \\
334)\end{array}$ & $\begin{array}{l}282.5 \quad(239- \\
382)\end{array}$ & $\begin{array}{l}284.0 \quad(248- \\
327)\end{array}$ & 0.774 \\
\hline Leukocytes, $10^{9} / 1$ & $\begin{array}{ll}7.0 & (4.0- \\
19.2)\end{array}$ & $\begin{array}{l}8.81 \\
18.3)\end{array}$ & $\begin{array}{l}11.95 \\
23.7)\end{array}$ & $7.9(6-10.3)$ & $0.006^{*}$ \\
\hline Platelets, $10^{9} / 1$ & $\begin{array}{l}316 \\
424)\end{array}$ & $365(63-554)$ & $\begin{array}{l}381 \\
724)\end{array}$ & $\begin{array}{l}329 \quad(158- \\
450)\end{array}$ & 0.091 \\
\hline Erythrocytes, $10^{12} / 1$ & $4.7(4-5.3)$ & $4.68(2.8-5.2)$ & $5.12(4.1-6)$ & $4.7(4.1-5.2)$ & 0.128 \\
\hline Hemoglobin, g/l & $\begin{array}{l}129 \\
152)\end{array}$ & 115 (91-154) & $113(53-148)$ & $\begin{array}{l}132 \quad(120- \\
150)\end{array}$ & $0.027 *$ \\
\hline $\mathrm{MCV}, \mathrm{fl}$ & $\begin{array}{ll}86.3 & (79.1- \\
97.2) & \\
\end{array}$ & $\begin{array}{ll}83.2 & (72.4- \\
105.3) & \\
\end{array}$ & $\begin{array}{ll}76.3 & (57.8- \\
97) & \\
\end{array}$ & $\begin{array}{ll}82.5 & (78.5- \\
87.3) & \\
\end{array}$ & $0.001 *$ \\
\hline MCH, g/l & $\begin{array}{l}27.9 \quad(23.9- \\
32)\end{array}$ & $\begin{array}{l}24.8 \\
28.2)\end{array}$ & $\begin{array}{l}21.4 \\
30.6)\end{array}$ & $\begin{array}{l}28.1 \quad(27.2- \\
29.7)\end{array}$ & $0.001^{*}$ \\
\hline MCHC, g/l & $\begin{array}{l}320 \\
343)\end{array}$ & $\begin{array}{ll}312 & (254- \\
273) & \\
\end{array}$ & $\begin{array}{ll}290 & (268- \\
319) & \\
\end{array}$ & $\begin{array}{ll}342 & \text { (328- } \\
352) & \\
\end{array}$ & $0.000 *$ \\
\hline
\end{tabular}

Note. * - differences significant at $\mathrm{p}<0.05$. All abbreviations are given in the main body.

We noted that blood serum transferrin and ferritin levels in NUC patients did not differ from the control group parameters significantly during the analysis of serum parameters. We established increase in the level of serum inflammatory response markers, in direct correlation with the increase in the inflammation activity degree at that. At the same time, we also noted reduction in hemoglobin and iron levels (pic. 3) - in direct correlation with the inflammation activity degree increase $(p<0.05)$. Thus the most significant inflammatory response markers are IL 6 and hepcidin. 
Pic. 2. Mean corpuscular hemoglobin concentration (MCHC, g/l) parameter indices at different degrees of activity of nonspecific ulcerative colitis.

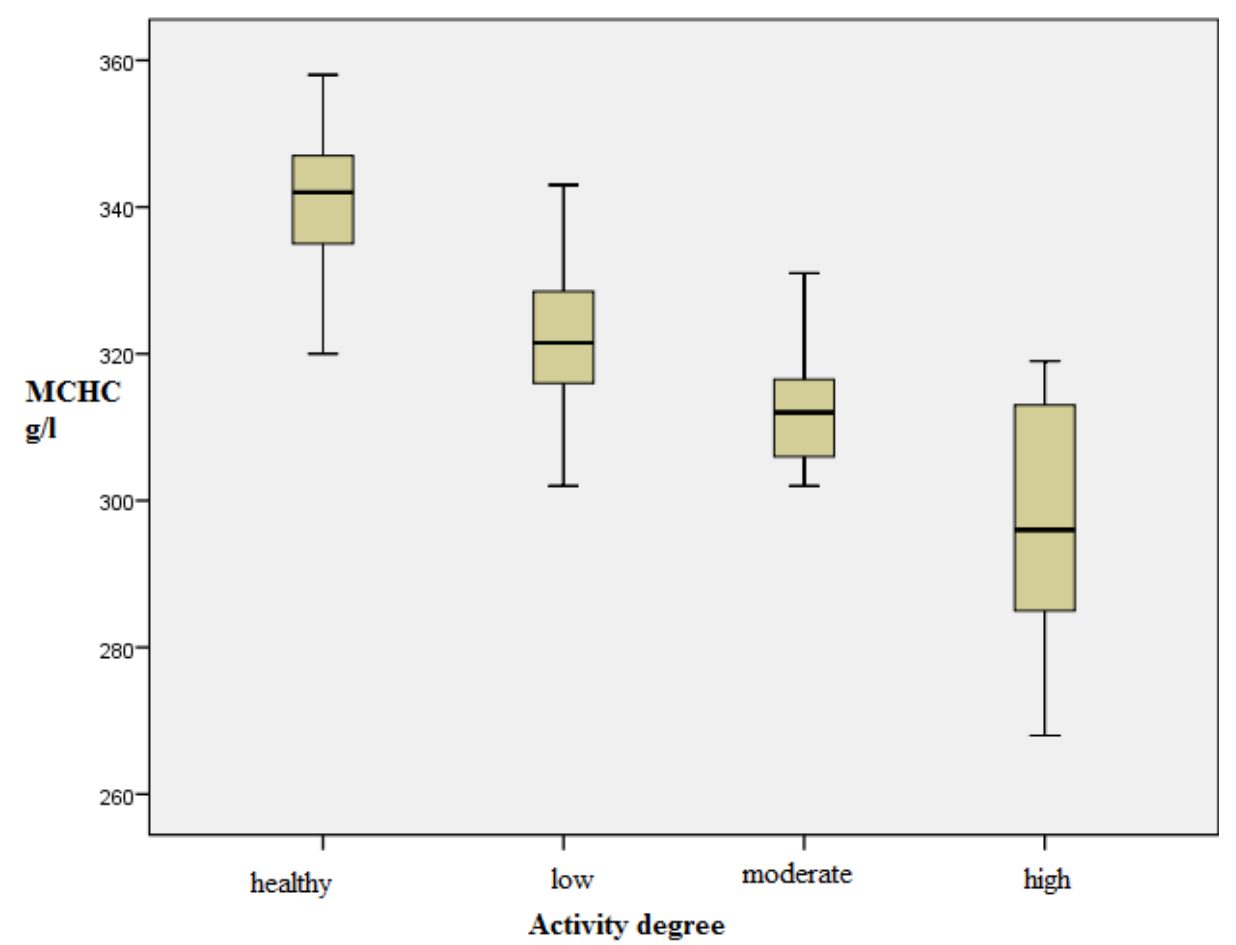

Pic. 3. Serum iron level $(\mathrm{mcmol} / \mathrm{l})$ at different degrees of activity of nonspecific ulcerative colitis.

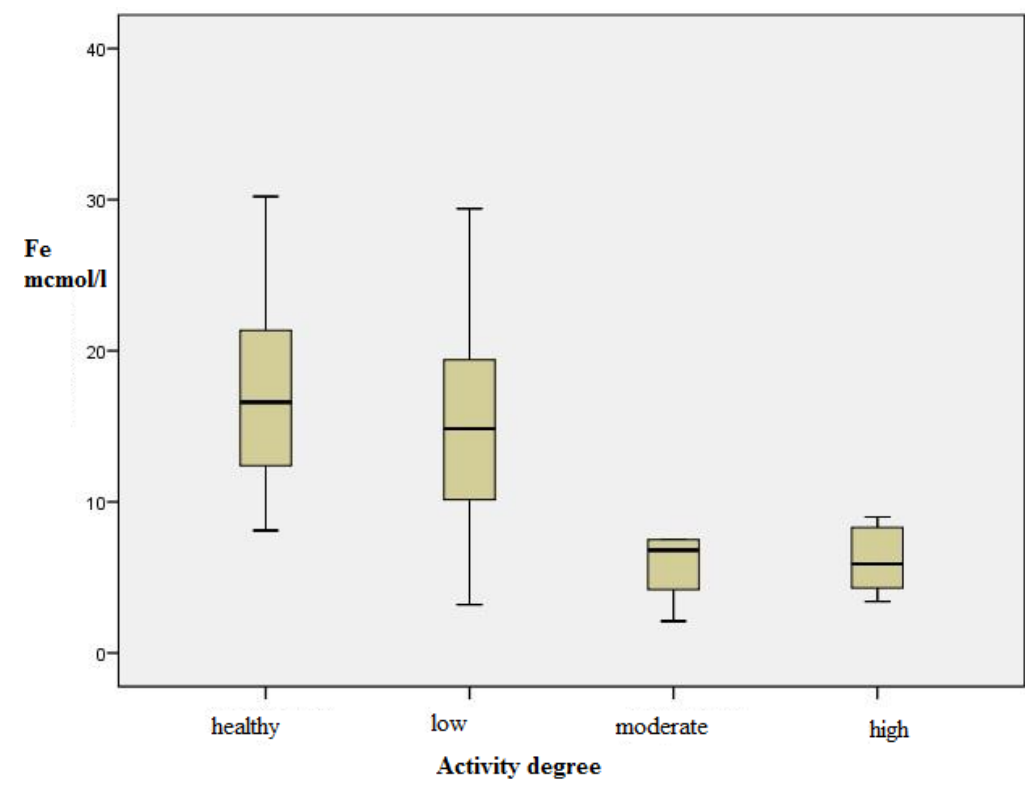

\section{Discussion}

According to the trial, levels of platelets and erythrocytes do not significantly alter in children with NUC. There is moderate direct Spearman correlation between IL 6 and CRP in case of high $(\mathrm{r}=0.55, \mathrm{p}<0.05)$, moderate $(\mathrm{r}=0.8, \mathrm{p}<0.05)$ and low $(\mathrm{r}=0.47, \mathrm{p}<0.05)$ activity; between hepcidin and IL 6 - in case of moderate and high activity $(\mathrm{r}=0.70, \mathrm{p}<0.05)$. Inverse correlation was revealed between levels of CRP and iron in case of high inflammation activity degree $(\mathrm{r}=-0.6$; $\mathrm{p}<0.05$ ) and lack of such correlation in other groups; between IL 6 and iron - only in case of 
high activity ( $\mathrm{r}=-0.46 ; \mathrm{p}<0.05)$; between hepcidin and iron - in case of moderate and high activity ( $\mathrm{r}=-0.55 ; \mathrm{p}<0.05)$. Thus, hepcidin is capable of reflecting inflammatory alterations better than other parameters even if the disease activity is low (pic. 4), unlike a well-known parameter CRP, which is unable to fully reflect degree of inflammation activity. Given the data obtained during the detailed examination of patients with NUC, we established that this disease causes peripheral hemopoiesis alterations and that red blood indices are sensitive to inflammation with laboratory tests.

Pic. 4. Serum hepcidin level (ng/ml) t different degrees of activity of nonspecific ulcerative colitis.

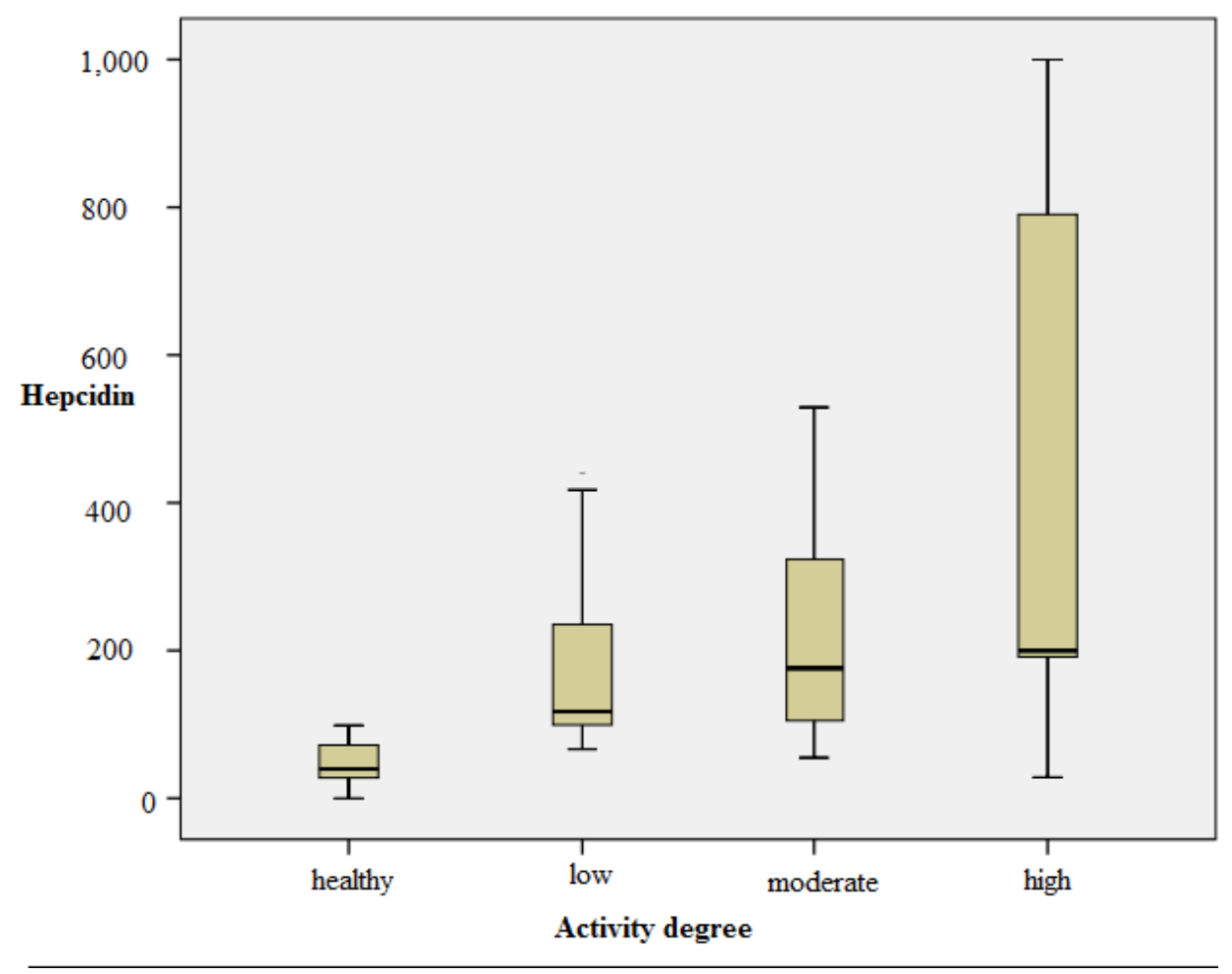

The obtained data may promote development of new approaches to inflammation activity determination at NUC and, possible, diagnostics of erythron disorders in the future [8]. We conducted comparative analysis of inflammation activity influence on hemopoiesis and evaluation of significance of inflammatory markers and hemopoiesis for clinical practice given their sensitivity and specificity.

We analyzed all tests and selected those, which are most sensitive to alterations in the setting of inflammation. We used such selection criteria as area under the curve (AUC), sensitivity and specificity of tests given the confidence interval 95\% (CI 95\%), as tests with AUC of 0.8-1.0 and high sensitivity are considered to be of the best quality. Tb. 2 presents comparative analysis of the most significant laboratory inflammatory markers.

Table 2. Results of comparative analysis of inflammatory response markers.

\begin{tabular}{|l|l|l|l|}
\hline Parameters & CRP (AUC) & IL 6 (AUC) & Hepcidin (AUC) \\
\cline { 1 - 2 } \multicolumn{1}{|c|}{ Disease activity } & & & \\
\hline Low & 0.397 & 0.724 & 0.84 \\
\hline Moderate & 0.644 & 0.99 & 0.87 \\
\hline
\end{tabular}

Note. AUC - area under the curve. 
According to the tb. 2 data, IL 6 and hepcidin have higher AUC index, therefore, they are capable of responding even to the slightest inflammatory alterations with a rather high level of specificity and sensitivity. CRP parameter may manifest itself with sufficient specificity only in case of moderate and high activity degree. Neither peripheral blood parameter features these data, which is why evaluation of peripheral blood parameters should not be conducted on a separate basis, but together with serum inflammatory response and inflammation activity degree markers.

\section{Conclusion}

Evaluation of laboratory parameters of inflammatory response and status of peripheral hemopoiesis at ulcerative colitis in children is an important issue of pediatrics, as singular laboratory tests are not capable of providing adequate approach to the evaluation of pathologic disorders in the body.

The selected diagnostically significant inflammatory response markers, such as hepcidin and IL 6 may be used as basic inflammation activity evaluation criteria; CRP - as an additional criterion. Among the peripheral blood parameters (erythrocyte indices), preference ought to be given to the MCHC parameter for evaluation of possible erythron system disorders.

\section{REFERENCES}

1. Aleksandrova Yu. N. Pediatriya - Peduatrics. 2007; 86 (3): 124-128.

2. Ketlinskii S. A., Simbirtsev A. S. Tsitokiny [Cytokines]. St. Petersburg, «Izdatel'stvo Foliant» LLC, 2008. 550 p.

3. Botvin'eva V. V., Gordeeva O. B., Namazova-Baranova L. S. Pediatricheskaya farmakologiya - Pediatric pharmacology. 2012; 9 (5): 35-40.

4. Dan Turner, Simon P. L. Travis, Anne M. Griffiths, Frank M. Ruemmele, Arie Levine, Eric I. Benchimol, Marla Dubinsky, George

Alex, Robert N. Baldassano, Jacob C. Langer, Robert Shamberger. The American Journal of Gastroenterology. 2011; 1038 (10): 574-588.

5. Briggs C. New red cell parameters on the Sysmex XE-2100 as potential markers of functional iron deficiency. Infus Ther Transf Med. 2001; 28: 256-258.

6. Ganz T. Hepcidin, a key regulator of iron metabolism and mediator of anemia of inflammation. Blood. 2003; 102: 783-788.

7. Titov V. N. Klinicheskaya laboratornaya diagnostika - Clinical laboratory diagnosis. 2008;

2: $3-14$.

8. Gordeeva O. B., Botvin'eva V. V., Namazova-Baranova L. S. Pediatricheskaya farmakologiya - Pediatric pharmacology. 2012; 9 (6): 110-112.

9. Namazova-Baranova L. S. Pediatricheskaya farmakologiya - Pediatric pharmacology. 2012; 9 (4): 15-24. 